\title{
Supercontinuum sources for multimodal MIR-OCT imaging
}

\author{
Ivan Zorin ${ }^{1}$, Jakob Kilgus ${ }^{1}$, Markus Brandstetter ${ }^{1}$, Rong Su${ }^{3}$, Bettina Heise ${ }^{1,2}$ \\ ${ }^{1}$ Research Center for Non-destructive Testing (RECENDT), 4040 Linz, Austria \\ ${ }^{2}$ Johannes Kepler University Linz (JKU), 4040 Linz, Austria \\ ${ }^{3}$ Manufacturing Metrology Team, Faculty of Engineering, University of Nottingham, Nottingham NG8 1BB, UK \\ Ivan.Zorin@recendt.at
}

\begin{abstract}
In this paper we present a multimodal OCT imaging system operating in the NIR and MIR spectral region. NIR/MIR Supercontinuum source based multimodal OCT provides new insights in scattering .materials. The system combines the modality of OCT and spectroscopy and enables a structural and specific characterization of different ceramics, polymers or composites. $\odot 2019$ The Author(s) OCIS codes: (110.0110) Imaging systems, (110.4500) Optical coherence tomography, (300.6340) Spectroscopy, infrared
\end{abstract}

\section{Introduction}

Recently the progress in Supercontiuum (SC) source developments has enables multiple new applications, for example in coherent and non-coherent imaging, in spectroscopy $[1,2]$ or for data transmission. While at the beginning these SC sources have been covered the near infrared (NIR) range, meanwhile they are advanced also covering the mid infrared (MIR) range. For Optical Coherence Tomography (OCT) imaging these light sources are beneficial due to their low temporal coherence. The higher spatial coherence is rather detrimental for OCT, in particular for full field OCT versions. However, there are also challenges which have to be tackled by the integration into a raster scanning OCT system. In particular noise fluctuations and repetition rates must be adapted regarding the scanning rate of the OCT system which defines finally the usability of different versions of these SC sources. - We will show here a SC based multimodal MIR OCT system applicable in the field of non-destructive testing (NDT).

\section{Measurements}

Before the operational assemblage of the OCT system the NIR/MIR-SC source has been characterized with respect to its noise floor and spectral features. The relative pulse energy noise (REN) of the SC source is a key parameter and was characterized for different repetition rates (varied with an external trigger) and power levels. The variance of a sequence of 1000 time-integrated pulses, normalized by the mean value has yielded the relative pulse energy noise level (REN) of the SC source [3]. The REN provides a measure of the wavelength averaged noise. The pulse forms were measured using a multiple junction photovoltaic HgCdTe detector (PVM, VIGO) with a time constant of $0.7 \mathrm{~ns}$ and then post-processed in Python. The spectral characterization of SC has been performed by Fourier transform infrared spectrometer and monochromator measurements.

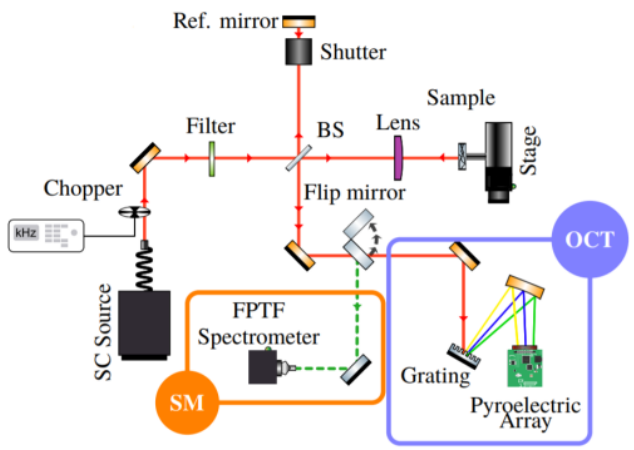

Figure 1: Scheme of the multimodal MIR Fourier Domain (FD) OCT system

The scheme of the (FD)-OCT system is depicted in Figure 1. As a multimodal system it consists of an OCT imaging modality and a spectroscopic modality (SM). For the first essential components are: the MIR SC source (based on ZBLAN fiber, $40 \mathrm{~mW}$ average power within OCT range), the OCT mirror based grating spectrometer (Czerny-Turner configuration), and the pyroelectric detector with synchronized chopper. For the spectroscopic part a microspectrometer (Fabry-Pérot tunable filter, FPTF) can be integrated, for instance enabled by a flippable mirror in the optical path. The optical setting has been simulated by ZEMAX, a comprehensive signal processing and aberration correction for the wide spectral range have been performed [4]. 
The FD-OCT system was also characterized and yields a sensitivity (SNR) of about $82 \mathrm{~dB}$, a lateral resolution of $35 \mu \mathrm{m}$ and an axial resolution around $40 \mu \mathrm{m}$. A spatial resolution, and effective probing depth for ceramic samples of up to $1.2 \mathrm{~mm}$ was achieved. The frame rate of the acquisition system is currently limited by $100 \mathrm{~Hz}$.

\section{Results}

First the multimodal MIR OCT system has been exemplified by standard test pattern, like alumina and zirconia plane plates. Later our system was tested for more complicated and composed material systems, like paint and varnish samples for ceramic composites. The layered structures of the ceramic composites could be confirmed by MIR FD-OCT, while a comparable measurement by NIR OCT system failed for recognizing internal details in ceramics specimen, as shown in Figure 2.
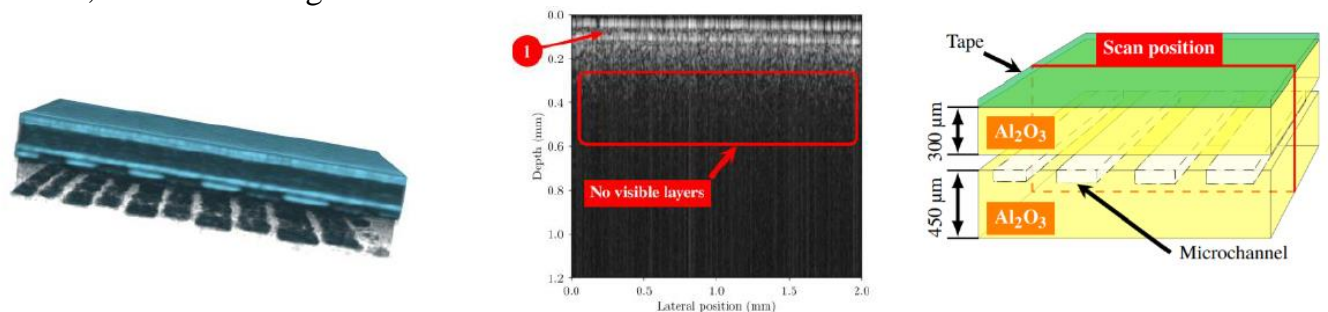

Figure 2: (left) 3D OCT volume stack of a composite ceramic structures, measured by MIR FD-OCT; (middle) cross-sectional OCT image measured by commercial NIR FD-OCT system, no internal structures are visible, and (right) the $3 D$ model of the tested ceramic structures. The imaging volume for the $3 D$ MIR OCT stack is $10 \times 10 \times 1.2 \mathrm{~mm}$

Furthermore, our advanced MIR FD-OCT also has been demonstrated for imaging in thicker (but weak absorbing) polymer components, e.g. IC cards. Here the hidden, embedded structures, like metallic antennas/electrodes have been visualized through the polymer material, as depicted in the Figure 3.
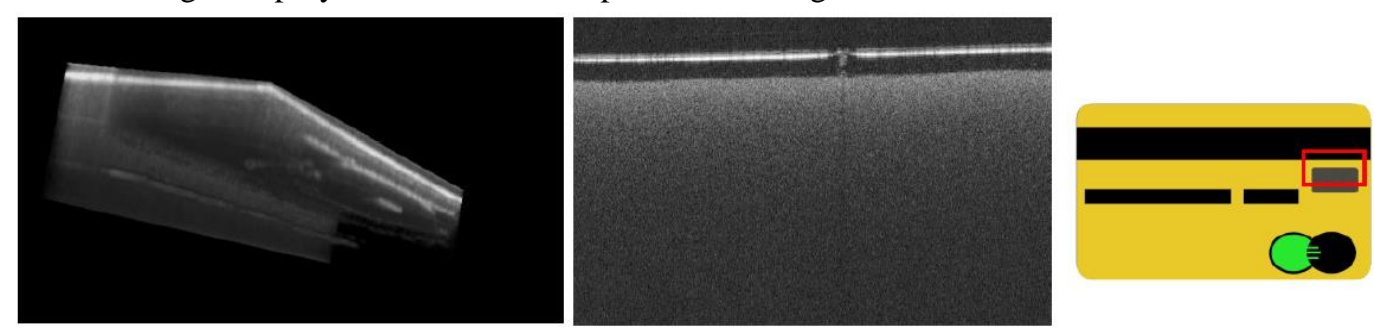

Figure 3: (left) 3D OCT volume stack of a polymer IC card with integrated chip, measured by MIR FD-OCT; (middle) cross-sectional OCT image measured by commercial NIR FD-OCT system, again no internal structures are visible, and (right) scheme of tested chip card. The imaging volume for the 3D MIR OCT stack is $12 x 8 \times 1.2 \mathrm{~mm}$

\section{Conclusions and Outlook}

Summarizing, we have illustrated an elegant multimodal MIR FD-OCT system with pyroelectric line array as detector. We have demonstrated this system successfully for NDT applications of ceramics and polymer composite structures. The SC source enables to discover novel spectral ranges for OCT imaging and probing. We also highlight the spectroscopic imaging features of the multimodal system as well. The OCT system can still be improved by advances in the pyroelectric detector controlling system resulting in an even higher sensitivity for future.

Prospectively, novel applications schemes for characterization of thin artificial teeth components or ceramic coated prosthetics, or for use in art (porcelain) conservation can be envisaged.

We acknowledge project funding by EU SUPUIR program and the Austrian national MICI and FTI research project.

\section{References}

[1] J. Kilgus, et al., "Diffraction limited mid-infrared reflectance microspectroscopy with a supercontinuum laser", Opt.Express 26, 30644-30654, (2018).

[2] Manoj K. Dasa, et al., "High pulse energy supercontinuum laser for spectroscopic optical resolution photoacoustic imaging of lipids in the 1650-1850 nm window," Biomed. Opt. Exp. 9(4), 1762-1770, (2018).

[3] B. Gonzalo et al., "Ultra-low noise SC source for ultra-high resolution OCT at $1300 \mathrm{~nm}$ ", SPIE 104860, (2018).

[4] I. Zorin et al., "MIR FD-OCT with a pyroelectric linear array”, Opt.Express 26 (25), 33428-33439, (2018). 